\title{
Some Chemical Aspects of Nutrition
}

$\mathrm{T}^{\mathrm{H}}$ HE thirty-fifth Bedson Lecture was delivered by Prof. J. C. Drummond on November 20 at Armstrong College, Newcastle-on-Tyne, on "Chemical Aspects of some Modern Nutritional Problems".

Prof. Drummond pointed out that animals are dependent on plants for certain compounds which they cannot synthesize themselves from potentially suitable material. Such compounds can be divided into two types: tissue-building stores, and what might be termed 'chemical lubricants', for example, vitamins and hormones.

Cystine is a good example of the former type. Animals cannot synthesize this important amino-acid even if provided with sulphur, in various forms, and closely related compounds not containing sulphur, for example, serine. However, they can use menthionine, and dithioldilactic acid, but not homocystine and cystineamine to replace a cystine deficiency. Persons suffering from cystinuria who are given menthionine excrete it as cystine only.

Vitamins $A, B_{1}, C$ and $D$ are examples of the second type. In dealing with the work leading to the synthesis of vitamin $\mathrm{B}_{1}$, Prof. Drummond pointed out that alteration in the position of the methyl group in the pyrimidine ring leads to the formation. of biologically inactive products.

Vitamin $\mathrm{A}$ has been shown to be a $\mathrm{C}_{20}$ compound closely related to the carotinoid pigments. Those carotinoids possessing a $\beta$-ionone ring and a suitable side chain act as precursors of the vitamin. $\beta$-carotene itself is probably quantitatively converted into it. It is remarkable that the molecule divides at the central double bond considering that polyene chains are usually susceptible to oxidative attack at various points. Certain birds can use xanthophylls as vitamin A precursors, whereas other animals cannot.
This suggests that there may be more than one vitamin $\mathrm{A}$.

Chemical studies of vitamin $\mathrm{C}$ ( $l$-ascorbic acid) are fairly complete, and it is interesting to compare the antiscorbutic activities of various substituted ascorbic acids. $d$-Ascorbic acid is quite inactive, $d$-araboascorbic acid possesses one twentieth the activity, while $l$-rhamno-ascorbic acid and dehydro-ascorbic acid are less active. The preparation of these synthetic products possessing activity raises the question of the possibility of the synthesis of compounds of greater antiscorbutic power than vitamin C.

Turning to vitamin D (calciferol), Prof. Drummond said that it is generally held that rupture of one ring of its sterol precursor takes place during irradiation with ultra-violet light. Vitamin $\mathrm{D}$ activity is not dependent on the presence of a double bond in the side chain. Recent work on the antirachitic substance produced by irradiation of 7.dehydrocholesterol and on the products formed when 7 . dehydrostigmasterol and 22.dihydro.7.dehydrostigmasterol are similarly treated was reviewed. The relation between constitution and biological action is not yet clearly apparent. There are two views to account for the activity of the various compounds. Either it is necessary to provide substances capable of being broken down to simpler molecules, or compounds with the same carbon skeleton as vitamin $D$ are required. The former gleans some support from the fact that compounds, very different in structure from those obtained from natural sources, are capable of producing œstrus. For example, $l$-keto-tetrahydro phenanthrene and certain dibenzanthrone diols have some activity. A more remarkable case is that reported by Prof. E. C. Dodds, who found that dihydroxydiphenyl is active.

\section{The Part played by Skin-Friction in Aeronautics*}

$\mathrm{T}$ HIS paper, running to some forty-seven pages of closely printed matter, is a book in itself. It is illustrated by twenty-seven figures, and is in five parts ; Part I deals with resistance due to skinfriction, on an empirical basis, the determinations of Froude and of the many workers who followed him being presented in the form of a logarithmic diagram, in which, as is usual, ordinates give values of the constant $\mathbf{C}_{0}$ (as in the expression $R=\mathrm{C}_{0} \rho V^{2} \times 2 a$ ) and abscissæ give Reynolds' numbers from $10^{2}$ to $10^{10}$; these numbers, denoted by the symbol $N_{R}$, cover the whole range from that proper to laminar flow, to that which obtains for ocean-going liners. The author points out that it is not possible to reconcile the wide variations in known data and gives a graph as indicating the probable minimum under ordinary average conditions. After a general discussion, an example is taken in the Graf Zeppelin; the hull resistance is calculated

* Synopsis of a paper by Dr. F. W. Lanchester, F.R.S., read before the Royal Aeronautical Society on November 12 on the basis suggested by Froude in the case of ships ; the horse-power is deduced for a flight speed of 80 m.p.h. and is in approximate agreement with that declared. A second example is given.

Parts II and III give in an abridged form the author's cyclic or vortex theory (sometimes referred to as the Prandtl theory) of sustentation, describing the two methods of treatment adopted by him in 1907 and 1914 respectively, and proving their quantitative identity. The criterion is shown to be the 'peripteral area' which defines the quantity of air handled by an aerofoil or the wings of a machine in flight. According to both methods the peripteral area is shown to be equal to 0.78 of the square of the span ; that is to say, the area of a circle having a diameter equal to the span. From this the aerodynamic resistance may be calculated making use of the Newtonian principle of the equality of force and momentum per second. Parts II and III, though not directly concerned with skin-friction, lead up to Part IV, in which it is shown that the theory of 